\title{
Piezophototronic Solar cell based on Third Generation Semiconductor Materials
}

\section{MICHAEL GYAN ( $\nabla$ mgyan173@gmail.com )}

UESTC: University of Electronic Science and Technology of China https://orcid.org/0000-0001-63372205

\section{Joseph Parbey}

UESTC: University of Electronic Science and Technology of China

FRANCIS BOTCHWEY

UESTC: University of Electronic Science and Technology of China

\section{Research Article}

Keywords: Third generation semiconductor, Piezophototronics, Piezotronics, Piezoelectricity, Solar cell

Posted Date: February 26th, 2021

DOI: https://doi.org/10.21203/rs.3.rs-256520/v1

License: (9) This work is licensed under a Creative Commons Attribution 4.0 International License. Read Full License 


\title{
Piezophototronic Solar cell based on Third Generation Semiconductor Materials
}

\author{
Michael Gyan ${ }^{\mathrm{a}, *}$, Joseph Parbey ${ }^{\mathrm{b}, \mathrm{c}}$, and Francis E. Botchey \\ ${ }^{a}$ School of Physics, University of Electronic Science and Technology of China, Chengdu 610054, China \\ ${ }^{b}$ School of Material Science, University of Electronic Science and Technology of China, Chengdu 610054, \\ China \\ ${ }^{c}$ Koforidua, Technical University, Ghana \\ *Corresponding Author: mgyan173@gmail.com; phone: +8613228202349
}

\begin{abstract}
By applying the outward uniform strain on the non-centrosymmetric piezoelectric semiconductor, the polarization charges on the material surface are induced. Polarization charges are often generated within the crystals provided that the applied strain is non-uniform. The strain applied has an effect on electronic transport and can be utilized to modulate the properties of the material. The effect of multiway coupling between piezoelectricity, semiconductor transport properties, and photoexcitation results in piezophototronic effects. Recent studies have shown the piezoelectric and semiconductor properties of thirdgeneration semiconductors have been used in photodetectors, LEDs, and nanogenerators. The thirdgeneration piezoelectric semiconductor can be used in high-performance photovoltaic cells. A thirdgeneration piezo-phototronic solar cell material is theoretically explored in this manuscript on the basis of a GaN metal-semiconductor interaction. This study aims to determine the effects of piezoelectric polarization on the electrical performance characteristics of this solar cell material. Performance parameters such as Power Conversion Efficiency, Fill Factors, I-V Characteristics, Open Circuit Voltage, and Maximum Output Power have been evaluated. The piezophototronic effect can enhance the open-circuit current voltage by 5.5 percent with an externally applied strain by 0.9 percent. The study will open a new window for the next generation of high-performance piezo-phototronic effects.
\end{abstract}


KEYWORDS: Third generation semiconductor, Piezophototronics, Piezotronics, Piezoelectricity, Solar cell

\section{Introduction}

Piezo-phototronics was initially proposed in 2010 [1-3]. The field of piezotronics has developed the study of the coupling between the semiconductor and piezoelectric characteristics for materials that concurrently exhibit semiconductor, photoexcitation, and piezoelectric characteristics. Also, the well-known field of optoelectronics studies the pairing of semiconductor properties with photoexcitation properties[4, 5]. The field of piezo-photonics was determined by the analysis of the linkage between piezoelectric characteristics and the characteristics of photo-excitation. The field of piezoelectric optoelectronics, which was the basis for the development of the new piezophototronics, was further developed by working on the coupling of semiconductor, photoexcitation, and piezoelectric properties[6-8]. The central feature of the piezophototronic effect is the use of piezoelectric potential to control the generation, separation, transport, and recombination processes of carriers at interfaces or junctions[1, 9]. High-efficiency optoelectronic devices, including solar cells, LEDs, and photodetectors, can be achieved by piezophototronic effects[6, $10-12]$.

By Comparing first-generation semiconductors, (for example, silicon, germanium) with the second-generation semiconductors, (for example, gallium arsenide, indium antimonide), the thirdgeneration semiconductor materials such as silicon carbide $(\mathrm{SiC})$, zinc oxide $(\mathrm{ZnO})$, gallium nitride $(\mathrm{GaN})$ and cadmium sulfide $(\mathrm{CdS})$ as a rule have wider bandgap, higher thermal conductivity, greater electron saturation rate and better radiation resistance properties, and in this way attract a lot of considerations in high temperature and high-frequency applications in recent years $[1,13,14]$. The greater part of the third-generation semiconductors is wurtzite structures, 
which have piezoelectric effects because of their absence of symmetry in certain directions[2 14,15]. These characteristics fill in as a decent scaffold for transferring mechanical stress signals between the adaptable semiconductor electronic device and the surrounding environment or the host (e.g., the human body)[16,17].

Third generation semiconductor materials, such as GaN and SiC, distinguished by a wide band gap, have attracted considerable interest in emerging consumer electronics, $5 \mathrm{G}$ telecommunication technologies, automated vehicles, optoelectronics and defense technology applications owing to its superior material properties, including high voltage resistance, high switching frequency, Hightemperature tolerance and high radiation resistance[18]. The wide bandgap nature and the good piezoelectric properties of these materials indicate that piezotronic and piezophototronic couplings may be important, providing excellent platforms for the analysis of the fundamental coupling between the piezoelectricity and a variety of interesting processes such as high-frequency transmission, high-field activity, and two-dimensional (2D) electron gas in associated system structures[19].

In this manuscript, we present the performance of third-generation semiconductor solar cells using a piezophototronic effect. The study model is shown below in Figure 1. A third-generation semiconductor, such as GaN, is sandwiched between two metal electrodes on the substrate as seen in Figure 1(a). One part of the metal-semiconductor-metal unit is the solar cell and the other is the electrode (ohmic contact) so it has an opposite output voltage. Polarization charges are added to the interface of the solar cell through the application of external strain as seen in Figures 1(b) and (c). The piezoelectric field raises or decreases the height of the Schottky barrier[20-22] as seen below. 
2. Modulation of Piezophototronics solar cell

The theoretical models are optimized for $\mathrm{p}-\mathrm{n}$ solar junction cells, and identical mathematical analyzes can be obtained for metal-semiconductor solar cells[23]. Shockley's theory is now employed to calculate the $\mathrm{I}-\mathrm{V}$ features of the piezoelectric dependent $\mathrm{p}$ -n junction, and evaluate the ideal p-n junction dependent on the aforementioned hypotheses: (1) the piezoelectric semiconductor has not degenerated in such a manner that the Boltzmann approximation can be implemented. (2) The piezoelectric p-n junction has an unstable depletion layer. (3) There is no generation and recombination present in the depletion layer, and the hole and electron currents are stable in the p-n junction. (4) The concentration of the dominant carrier is significantly greater than that of the minority injected. The total current density of the p-n solar cell is given by[24]:

$$
J=J_{o}\left[\exp \left(\frac{q V}{K T}\right)-1\right]-J_{s c}
$$

Here $J_{0}$ is the saturation current, $J_{s c}$ denotes the short-circuit current density, $k$ stands for the Boltzmann constant, $\mathrm{T}$ represents the temperature, $\mathrm{q}$ is the elementary charge and $\mathrm{V}$ is the applied voltage. The saturation current can be achieved by[24]:

$J_{o}=\frac{q D_{p} p_{n o}}{L_{p}}+\frac{q D_{n} n_{p o}}{L_{n}}$

where $L_{n}$ and $L_{p}$ are the diffusion lengths of holes and electrons, respectively; $n_{p o}$ and pno are the electron concentration inside the p-type semiconductor and the hole concentration inside the ntype semiconductor at thermal equilibrium, respectively. Thus, the intrinsic carrier density ni can be calculated as

$$
n_{i}=N_{c} \exp \left[-\frac{E_{c}-E_{i}}{K T}\right]
$$


Here $E_{i}$ is an underlying the intrinsic Fermi level, $N_{C}$ is the effective density of states in the conduction band, and $\mathrm{E}_{\mathrm{C}}$ is the bottom edge of the conduction band. Without a piezo potential at the interface of the p-n junction, the relationship between the saturated current density $\left(\mathrm{J}_{\mathrm{C} 0}\right)$ and Fermi Level (Е户) can be articulated as

$$
J_{c o}=\frac{q D_{p} n_{i}}{L_{p}} \exp \left(\frac{E_{i}-E_{F o}}{K T}\right)
$$

The Fermi Level with piezo-potential at the interface of a p-n junction is determined by

$$
E_{F}=E_{F o}-\frac{q^{2} \rho_{\text {piezo }} W_{\text {piezo }}^{2}}{2 \varepsilon_{s}}
$$

By adding equation 3, 4, and 5 the $\mathrm{I}-\mathrm{V}$ characteristics of the Piezophototronic solar cell based on a third-generation semiconductor can be derived as[25]

$$
J=J_{o} \exp \left[\frac{q^{2} \rho_{\text {piezo }} W_{\text {piezo }}^{2}}{2 \varepsilon_{s} K T}\right]\left[\exp \left(\frac{q V}{K T}\right)-1\right]-J_{s c}
$$

From Eqn. 6, the current transport through the p-n junction is a function of the piezoelectric charges, whose sign depends on the direction of the strain. Thus, both the magnitude and sign of the external strain (tensile or compressive) can be used to effectively adjust or control the transport current. The open-circuit voltage $\left(V_{o c}\right)$ can be evaluated by

$$
V_{o c}=\frac{K T}{q}\left[\operatorname{In}\left(\frac{J_{s c}}{J_{c o}}\right)+\frac{q^{2} \rho_{\text {piezo }} W_{\text {piezo }}^{2}}{2 \varepsilon_{s} K T}\right]
$$

The piezo-phototronic modulation ratio of the PSC can be described in terms of the open-circuit voltage and other output performance obtained from the PSC [26]:

$$
\gamma=\frac{\frac{q^{2} \rho_{\text {piezo }} W_{\text {piezo }}^{2}}{2 \varepsilon_{s} K T}}{\operatorname{In}\left(\frac{J_{s c}}{J_{c o}}\right)}
$$


In addition, the output power is estimated as

$$
P(V)=V J(V) \approx V\left\{J_{o} \exp \left(\frac{q^{2} \rho_{\text {piezo }} W_{\text {piezo }}^{2}}{2 \varepsilon_{s} K T}\right)\left[\exp \left(\frac{q V}{K T}\right)-1\right]-J_{s c}\right\}
$$

The maximum voltage satisfies the following equation[27]:

$$
V_{m}+\frac{K T}{q} \operatorname{In}\left(\frac{q V_{m}}{K T}+1\right)=\frac{K T}{q}\left[\operatorname{In}\left(\frac{J_{s c}}{J_{c o}}\right)+\frac{q^{2} \rho_{\text {piezo }} W_{\text {piezo }}^{2}}{2 \varepsilon_{s} K T}\right]
$$

Consequently, $\mathrm{V}_{\mathrm{m}}$ varies with the piezoelectric charges which are induced by the applied strain $\varepsilon$.

The maximum current density can be obtained as:

$$
J_{m}=J_{o} \exp \left[\frac{q^{2} \rho_{\text {piezo }} W_{\text {piezo }}^{2}}{2 \varepsilon_{s} K T}\right]\left[\exp \left(\frac{q V_{m}}{K T}\right)-1\right]-J_{s c}
$$

The output maximum power $\mathrm{P}_{\mathrm{m}}$ can be estimated as

$$
P_{m}=V_{m} J_{m}
$$

The fill factor can be derived from the method described in reference[28],

$$
F F=\frac{J_{m} V_{m}}{J_{s c} V_{o c}} \approx \frac{P_{m}}{J_{s c} V_{o c}}
$$

The power conversion efficiency (PCE) is defined in $[22,28]$ :

$$
P C E=\frac{J_{s c} V_{o c} F F}{P_{i n}}
$$

\section{Result and Discussion}

Typical constants are utilized in computations of the performance parameters such as the Voc, Pm, PCE, and FF. The temperature was assigned at 300K, Wpiezo is thought to be $0.543 \mathrm{~nm}$ [29], the relative dielectric constant of $\mathrm{GaN}$ is 8.9 [30], and $\mathrm{e}_{33}$ of the $\mathrm{GaN}$ is estimated to be $0.73 \mathrm{C} / \mathrm{m}^{2}$ [31]. The relative current density $\mathrm{J} / \mathrm{J}_{\mathrm{pn} 0}$ versus voltage $\left(\mathrm{J} / \mathrm{J}_{\mathrm{pn} 0}-\mathrm{V}\right.$ curve $)$ of the GaN PSC with the external strain varying in the range of $-0.9 \%$ to $0.9 \%$ When the short circuit current $\mathrm{J}_{\mathrm{sc}}$ is taking to be 4.4 
$\mathrm{mA} / \mathrm{cm}^{2}[32]$ is plotted in Figure 2(a). Figure 2(b) shows the PCE of piezoelectric solar cells based on different types of materials at an external strain of $0.9 \%$, indicating that the $\mathrm{J}$ increases with the strain and peaks at Vm. By employing equation (13) and equation (14), the Fill Factor (FF) and power conversion efficiency (PCE) parameter which aid in explaining the characteristics and performance of PSC is illustrated in Figure 2(c)and (d). The FF is linearly dependent on the externally applied strain between the regions $-0.9 \%$ to 0.9 with a step of $0.3 \%$. The improvement in FF can be credit to the increased Voc when is under strain. The PCE and modulation ratio $(\gamma)$ considered in Figure 2 (b) and (c) is determined by utilizing similar parameters as in GaN such as $\mathrm{J}_{\mathrm{sc}}, \mathrm{J}_{\mathrm{pno}}$, etc, at an applied strain of $0.9 \%$ and $1 \%$ respectively. The different parameters utilized are the piezoelectric constant and the relative dielectric constant. The piezoelectric constant and relative dielectric constant are given in Table 1.1. The PCE and modulation ratio $(\gamma)$ of AlN is observed to increase more distinctly than $\mathrm{GaN}$ and $\mathrm{ZnO}$ within the strain region of 0.9 and $1 \%$ respectively. This can be attributed to the large piezoelectric constant and small relative dielectric constant of AlN material. By considering the impact of material properties, the ratio of piezoelectric constant to that of relative dielectric constant plays an essential part in the performance of PSC $[33,34]$. Among the third-generation semiconductor materials, the AlN has a noteworthy modulation ratio of $9.3 \%$ follows by $\mathrm{ZnO}(7.96 \%)$ and $\mathrm{GaN}(4.78 \%)$. The modulation ratio of AlN happens to be almost twice greater than GaN. The superior performance of $\mathrm{AlN}, \mathrm{ZnO}$, and $\mathrm{GaN}$ is due to the large piezoelectric constant, this demonstrates that a good performing material is the one with a large piezoelectric constant and small relative dielectric constant.

Figure 3(a) and (b) show the graph of maximum power $(\mathrm{Pm})$ and open-circuit voltage (Voc) against applied external strain. By utilizing Equation (7) and Equation (12), the Voc and Pm are linearly identifying with the strain(s). By introducing the piezo-phototronic effect, the performance 
parameters of the GaN PSC improve due to enhancement in Vm and Pm. The modulation ratio for GaN PSC as against $W_{\text {piezo }}$ as a function of strain is illustrated in Figure 3(c). As the width of the piezo-charge opens up the modulation ratio also increases. Furthermore, the modulation ratio is linearly dependent on both the external applied strain and $\mathrm{W}_{\text {piezo. The semiconductor material and }}$ metal contact can influence $\mathrm{W}_{\text {piezo }}[35,36]$. The piezoelectric constant and their dielectric constant of different third-generation semiconductor materials are plotted in Figure 3(d).

\section{Summary}

The idea of piezophototronic enhanced third-generation semiconductor material solar cells has been proposed and hence reenacted in this work. Key performance parameters portraying the device including Voc, Pm, P, and FF have been mathematically determined. It is indicated that the PSC shows an enhanced performance under externally applied strains, especially for the modulation ratio. Additionally, $\mathrm{GaN}, \mathrm{ZnO}$, and $\mathrm{AlN}$ show a more prominent potential for highefficiency PSCs. This gives physical insights into the PSCs and can serve as guidance on the design of third generation piezo-phototronic energy harvesting devices.

\section{Conflict of interest}

The authors declare that they have no conflict of interest

\section{Funding}

This research did not receive any specific grant from funding agencies in the public, commercial, or not-for-profit sectors

\section{Author contribution statement}

All authors have been contributed equally

\section{References}

[1] Z. L. Wang, "Piezopotential gated nanowire devices: Piezotronics and piezophototronics," Nano Today, vol. 5, no. 6, pp. 540-552, 2010, doi: 
https://doi.org/10.1016/j.nantod.2010.10.008.

[2] Z. L. Wang, W. Wu, and C. Falconi, "Piezotronics and piezo-phototronics with thirdgeneration semiconductors," MRS Bulletin. 2018, doi: 10.1557/mrs.2018.263.

[3] Y. Zhang, Y. Leng, M. Willatzen, and B. Huang, "Theory of piezotronics and piezophototronics," MRS Bull., 2018, doi: 10.1557/mrs.2018.297.

[4] X. Wen, W. Wu, C. Pan, Y. Hu, Q. Yang, and Z. Lin Wang, "Development and progress in piezotronics," Nano Energy, 2014, doi: 10.1016/j.nanoen.2014.10.037.

[5] Z. L. Wang, "The new field of nanopiezotronics," Mater. Today, 2007, doi: 10.1016/S1369-7021(07)70076-7.

[6] J. Hao and C. N. Xu, "Piezophotonics: From fundamentals and materials to applications," MRS Bull., 2018, doi: 10.1557/mrs.2018.296.

[7] M. C. Wong, L. Chen, M. K. Tsang, Y. Zhang, and J. Hao, "Magnetic-Induced Luminescence from Flexible Composite Laminates by Coupling Magnetic Field to Piezophotonic Effect," Adv. Mater., 2015, doi: 10.1002/adma.201502015.

[8] M. C. Wong, L. Chen, G. Bai, L. B. Huang, and J. Hao, "Temporal and Remote Tuning of Piezophotonic-Effect-Induced Luminescence and Color Gamut via Modulating Magnetic Field," Adv. Mater., 2017, doi: 10.1002/adma.201701945.

[9] Z. L. Wang, "Progress in piezotronics and piezo-phototronics," Advanced Materials. 2012, doi: 10.1002/adma.201104365.

[10] W. Wu and Z. L. Wang, "Piezotronics and piezo-phototronics for adaptive electronics and optoelectronics," Nat. Rev. Mater., vol. 1, no. 7, p. 16031, 2016, doi:

10.1038/natrevmats.2016.31.

[11] Y. Hu, C. Pan, and Z. L. Wang, "Recent progress in piezo-phototronics with extended materials, application areas and understanding," Semicond. Sci. Technol., 2017, doi: 10.1088/1361-6641/aa642e.

[12] R. Bao, Y. Hu, Q. Yang, and C. Pan, "Piezo-phototronic effect on optoelectronic nanodevices," MRS Bull., vol. 43, pp. 952-958, Dec. 2018, doi: 10.1557/mrs.2018.295.

[13] Z. L. Wang, "Nanopiezotronics,” Adv. Mater., 2007, doi: 10.1002/adma.200602918.

[14] C. Pan, J. Zhai, and Z. L. Wang, "Piezotronics and Piezo-phototronics of Third Generation Semiconductor Nanowires," Chemical Reviews. 2019, doi: 10.1021/acs.chemrev.8b00599.

[15] W. Wu and Z. L. Wang, "Piezotronics and piezo-phototronics for adaptive electronics and optoelectronics," Nature Reviews Materials. 2016, doi: 10.1038/natrevmats.2016.31.

[16] Z. L. Wang, J. Zhai, and L. Zhu, "Piezotronic and piezo-phototronic devices based on the third generation semiconductors," Chinese Sci. Bull., 2020, doi: 10.1360/tb-2019-0713.

[17] W. Sha, J. Zhang, S. Tan, X. Luo, and W. Hu, "III-nitride piezotronic/piezo-phototronic materials and devices," Journal of Physics D: Applied Physics. 2019, doi: 10.1088/13616463/ab04d6. 
[18] J. Millan, P. Godignon, X. Perpina, A. Perez-Tomas, and J. Rebollo, “A survey of wide bandgap power semiconductor devices," IEEE Trans. Power Electron., 2014, doi: 10.1109/TPEL.2013.2268900.

[19] X. Wang et al., "Piezotronic Effect Modulated Heterojunction Electron Gas in AlGaN/AlN/GaN Heterostructure Microwire," Adv. Mater., 2016, doi: 10.1002/adma.201601721.

[20] J. Fu, H. Zong, X. Hu, and H. Zhang, "Study on ultra-high sensitivity piezoelectric effect of GaN micro/nano columns," Nano Converg., 2019, doi: 10.1186/s40580-019-0203-4.

[21] G. Michael, G. Hu, D. Zheng, and Y. Zhang, "Piezo-phototronic solar cell based on 2D monochalcogenides materials," J. Phys. D. Appl. Phys., vol. 52, no. 20, p. 204001, Mar. 2019, doi: 10.1088/1361-6463/ab0ac4.

[22] G. Michael et al., "High-performance piezo-phototronic multijunction solar cells based on single-type two-dimensional materials," Nano Energy, vol. 76, Oct. 2020, doi: 10.1016/j.nanoen.2020.105091.

[23] Z. Dongqi, Z. Zhao, R. Huang, J. Nie, L. Li, and Y. Zhang, "High-performance piezophototronic solar cell based on two-dimensional materials," Nano Energy, vol. 32, pp. 448-453, Jan. 2017, doi: 10.1016/j.nanoen.2017.01.005.

[24] S. M. Sze and K. K. Ng, Physics of Semiconductor Devices. 2006.

[25] Y. Zhang, Y. Liu, and Z. L. Wang, "Fundamental theory of piezotronics," Adv. Mater., 2011, doi: 10.1002/adma.201100906.

[26] K. Gu, D. Zheng, L. Li, and Y. Zhang, "High-efficiency and stable piezo-phototronic organic perovskite solar cell," RSC Adv., vol. 8, no. 16, pp. 8694-8698, 2018, doi: 10.1039/C8RA00520F.

[27] Y. Wang, D. Zheng, L. Li, and Y. Zhang, "Enhanced Efficiency of Flexible GaN/Perovskite Solar Cells Based on the Piezo-Phototronic Effect," ACS Appl. Energy Mater., 2018, doi: 10.1021/acsaem.8b00713.

[28] G. Michael, G. Hu, D. Zheng, and Y. Zhang, "Piezo-phototronic solar cell based on 2D monochalcogenides materials," J. Phys. D. Appl. Phys., vol. 52, no. 20, 2019, doi: 10.1088/1361-6463/ab0ac4.

[29] W. Liu, A. Zhang, Y. Zhang, and Z. L. Wang, "Density functional studies on wurtzite piezotronic transistors: Influence of different semiconductors and metals on piezoelectric charge distribution and Schottky barrier," Nanotechnology, 2016, doi: 10.1088/09574484/27/20/205204.

[30] F. Schäffl, "Properties of advanced semiconductor materials : GaN, AlN, InN, BN, SiC, SiGe,” John Wiley Sons Inc New York, 2001, doi: citeulike-article-id:6144117.

[31] S. P. Wan, J. B. Xia, and K. Chang, "Effects of piezoelectricity and spontaneous polarization on electronic and optical properties of wurtzite III-V nitride quantum wells," J. Appl. Phys., 2001, doi: 10.1063/1.1413714. 
[32] C. Te Huang et al., "GaN nanowire arrays for high-output nanogenerators," J. Am. Chem. Soc., 2010, doi: 10.1021/ja909863a.

[33] G. Hu et al., "Enhanced performances of flexible $\mathrm{ZnO} /$ perovskite solar cells by piezophototronic effect," Nano Energy, vol. 23, pp. 27-33, 2016, doi:

https://doi.org/10.1016/j.nanoen.2016.02.057.

[34] J. Sun et al., "Piezo-phototronic Effect Enhanced Efficient Flexible Perovskite Solar Cells," ACS Nano, 2019, doi: 10.1021/acsnano.9b00125.

[35] W. Liu, A. Zhang, Y. Zhang, and Z. Lin Wang, "First principle simulations of piezotronic transistors," Nano Energy, 2015, doi: 10.1016/j.nanoen.2014.10.014.

[36] N. Suvansinpan, F. Hussain, G. Zhang, C. H. Chiu, Y. Cai, and Y. W. Zhang, "Substitutionally doped phosphorene: Electronic properties and gas sensing," Nanotechnology, 2016, doi: 10.1088/0957-4484/27/6/065708.

[37] F. Bernardini, V. Fiorentini, and D. Vanderbilt, "Spontaneous polarization and piezoelectric constants of III-V nitrides," Phys. Rev. B - Condens. Matter Mater. Phys., 1997, doi: 10.1103/PhysRevB.56.R10024.

[38] A. T. Collins, E. C. Lightowlers, and P. J. Dean, "Lattice vibration spectra of aluminum nitride," Phys. Rev., 1967, doi: 10.1103/PhysRev.158.833.

[39] Y. Zhang, J. Nie, and L. Li, "Piezotronic effect on the luminescence of quantum dots for micro/nano-newton force measurement," Nano Res., 2018, doi: 10.1007/s12274-0171814-x.

[40] Y. Zhang, Y. Yang, and Z. Wang, "Piezo-phototronics effect on nano/microwire solar cells,” Energy Environ. Sci., vol. 5, pp. 6850-6856, Apr. 2012, doi: 10.1039/C2EE00057A.

[41] D. Berlincourt, H. Jaffe, and L. R. Shiozawa, "Electroelastic properties of the sulfides, selenides, and tellurides of zinc and cadmium," Phys. Rev., 1963, doi: 10.1103/PhysRev.129.1009.

[42] P. E. Lippens and M. Lannoo, "Calculation of the band gap for small CdS and ZnS crystallites," Phys. Rev. B, 1989, doi: 10.1103/PhysRevB.39.10935.

[43] T. Wakaoka et al., "Confined synthesis of CdSe quantum dots in the pores of metalorganic frameworks," J. Mater. Chem. C, 2014, doi: 10.1039/c4tc01136h.

[44] I. Strzalkowski, S. Joshi, and C. R. Crowell, "Dielectric constant and its temperature dependence for GaAs, CdTe, and ZnSe,” Appl. Phys. Lett., 1976, doi: 10.1063/1.88755.

[45] G. A. Samara, "Temperature and pressure dependences of the dielectric constants of semiconductors," Phys. Rev. B, 1983, doi: 10.1103/PhysRevB.27.3494.

\section{Table}

Table 1:1 shows the piezoelectric constant and relative dielectric constant. 


\begin{tabular}{lcc}
\hline Material(s) & Piezoelectric constant $e_{33}\left({\left.\mathrm{c} / \mathrm{m}^{2}\right)}^{2}\right.$ & Relative dielectric constant \\
\hline GaN & $0.73[31]$ & $8.9[30]$ \\
AIN & $1.46[37]$ & $9.14[38]$ \\
InN & $15.3[37]$ & $0.97[30]$ \\
InAs & $15.15[37]$ & $-0.03[39]$ \\
ZnO & $1.22[40]$ & $8.92[40]$ \\
CdS & $5.7[41]$ & $0.44[42]$ \\
CdSe & $5.8[41]$ & $0.347[43]$ \\
CdTe & $11[37]$ & $0.03[44,45]$ \\
\hline
\end{tabular}

\section{Figure Captions}

Figure 1 Schematic structure and energy band configuration of third generation PSC semiconductor material (GaN). (a) Without strain; (b) with tensile strain; (c) with compressive strain;

Figure 2 (a). Relative current density-voltage $\left(\mathrm{J} / \mathrm{J}_{\mathrm{pn} 0}-\mathrm{V}\right)$ curve with Strain $(\varepsilon)$ increasing in the range of $[-0.9 \% 0.9 \%]$. (b) Comparative analysis of PCE for piezoelectric solar cell based on different types of materials with an external strain of $0.9 \%$. (c) $\gamma$ of the various types of piezoelectric solar cells material under an applied strain of $1 \%$ and (d) FF of GaN PSC with the applied strain varying in the region $[-0.9 \% 0.9 \%]$

Figure 3 (a) Pm and (b) Voc versus the external strain applied. (c) Modulation ratio $\gamma$ of the GaN PSC with $W_{\text {piezo }}$ under the strain $(\varepsilon)$ of $0.3 \% 0.6 \%$ and $1 \%$. (d) The piezoelectric constant of various third-generation semiconductor materials as opposed to their various dielectric constant. 
Figures
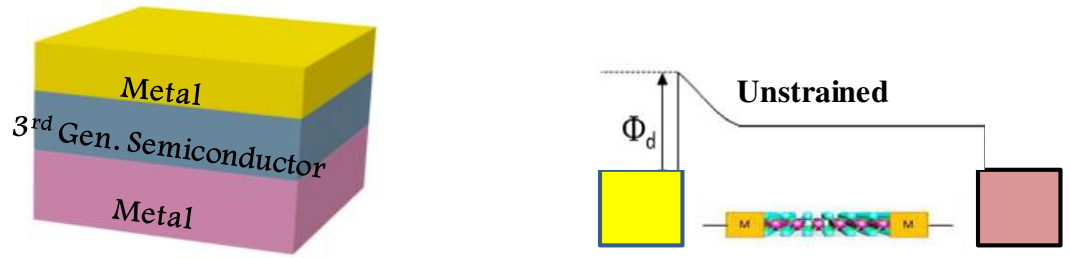

(a)
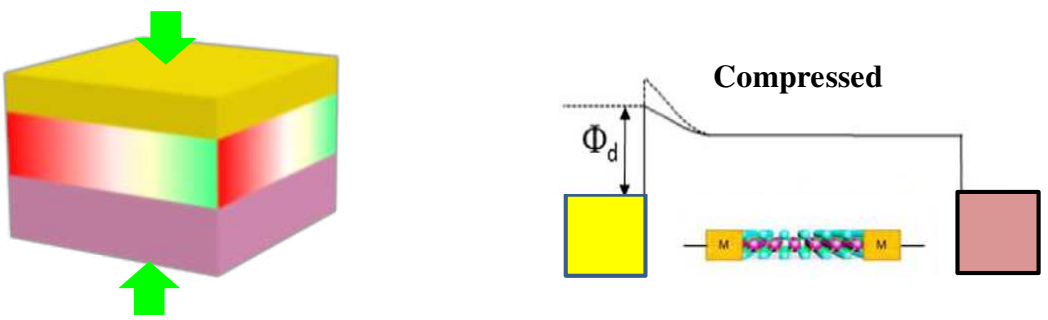

(b)
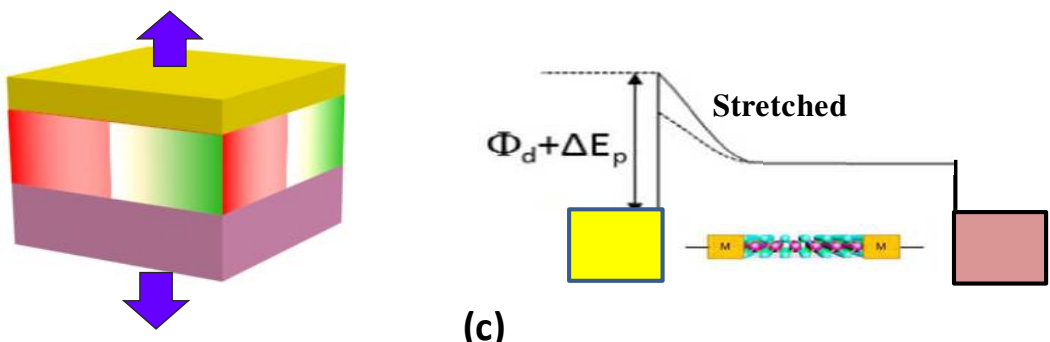

(c)

Figure 1 
(a)

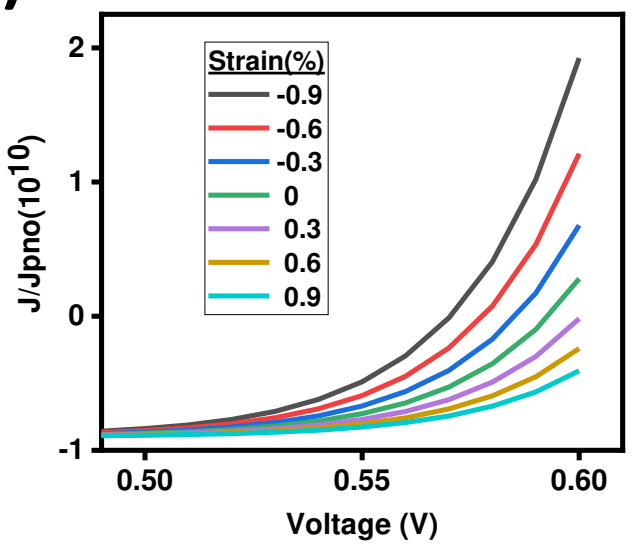

(c)

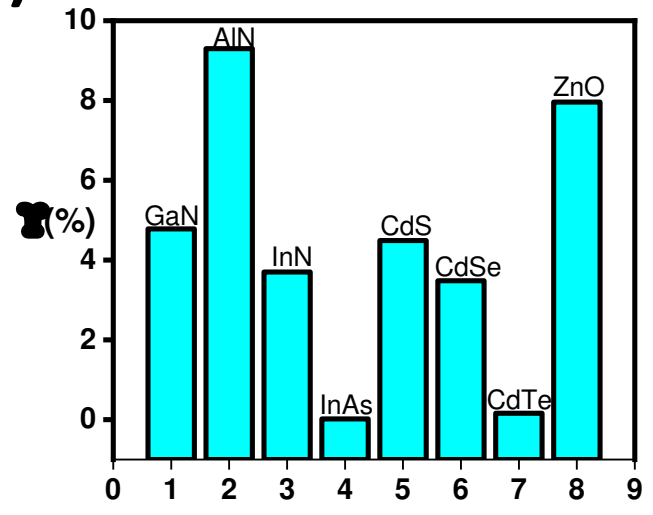

(b)

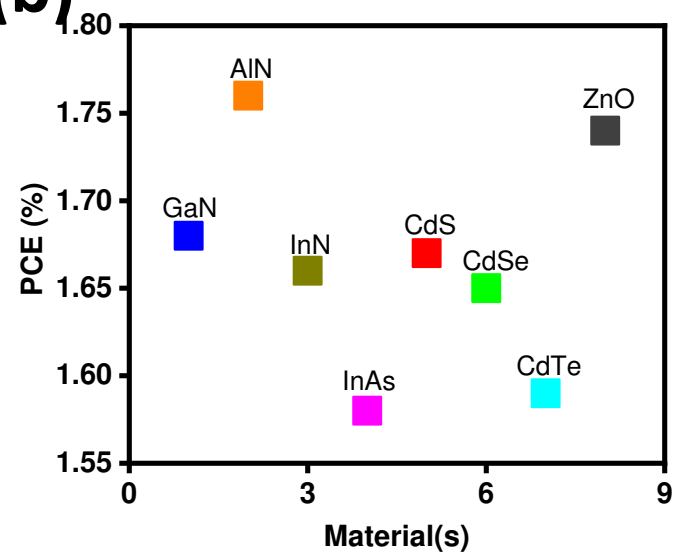

(d)

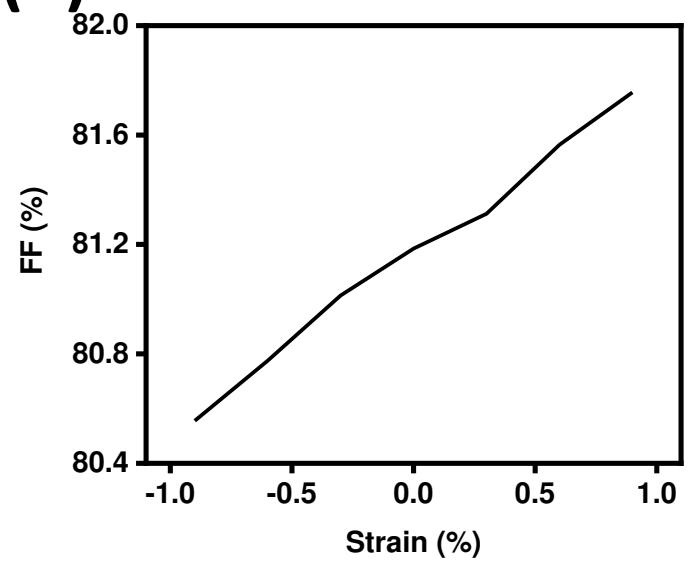

Figure 2 
(a)

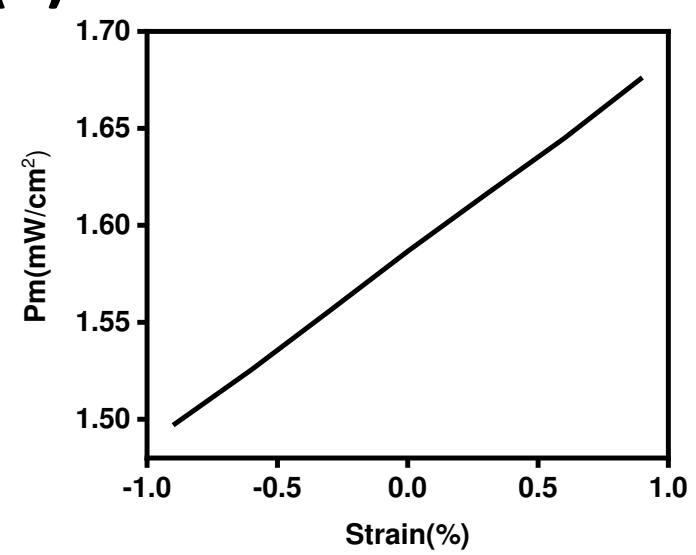

(b)

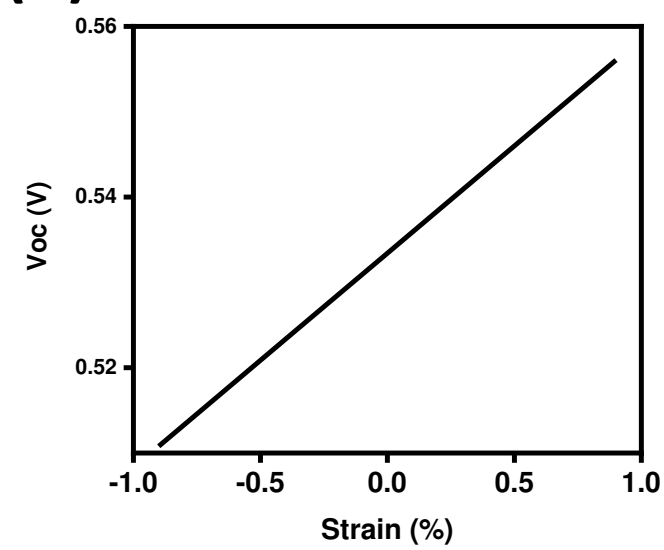

(c)

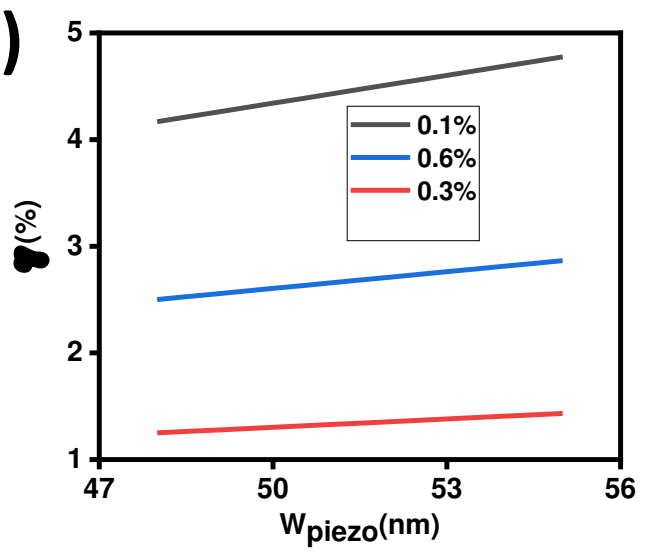

(d)

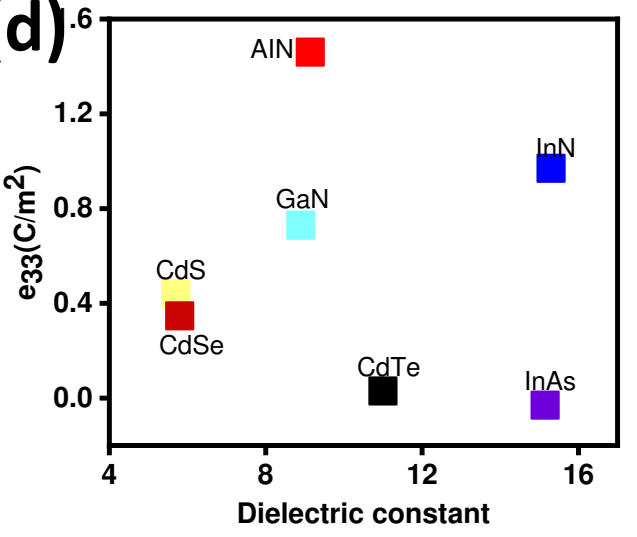

Figure 3 
Figures
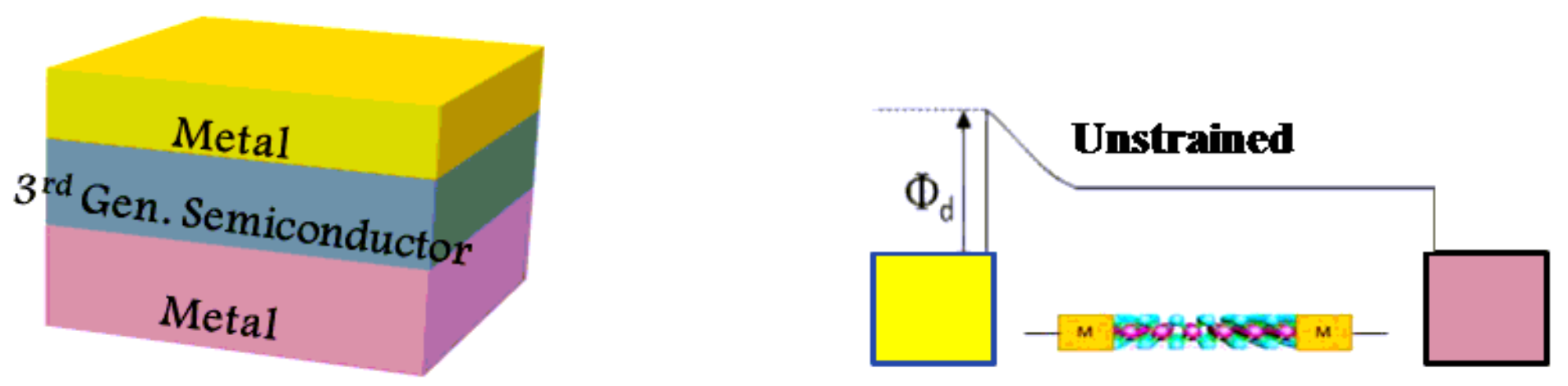

(a)
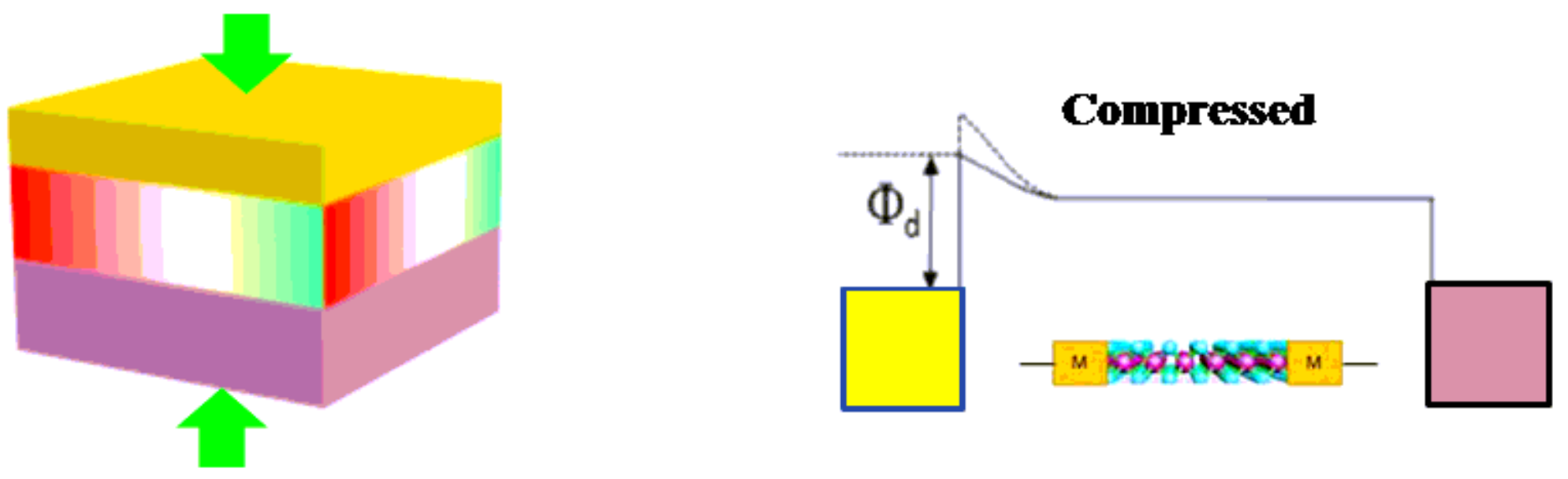

(b)
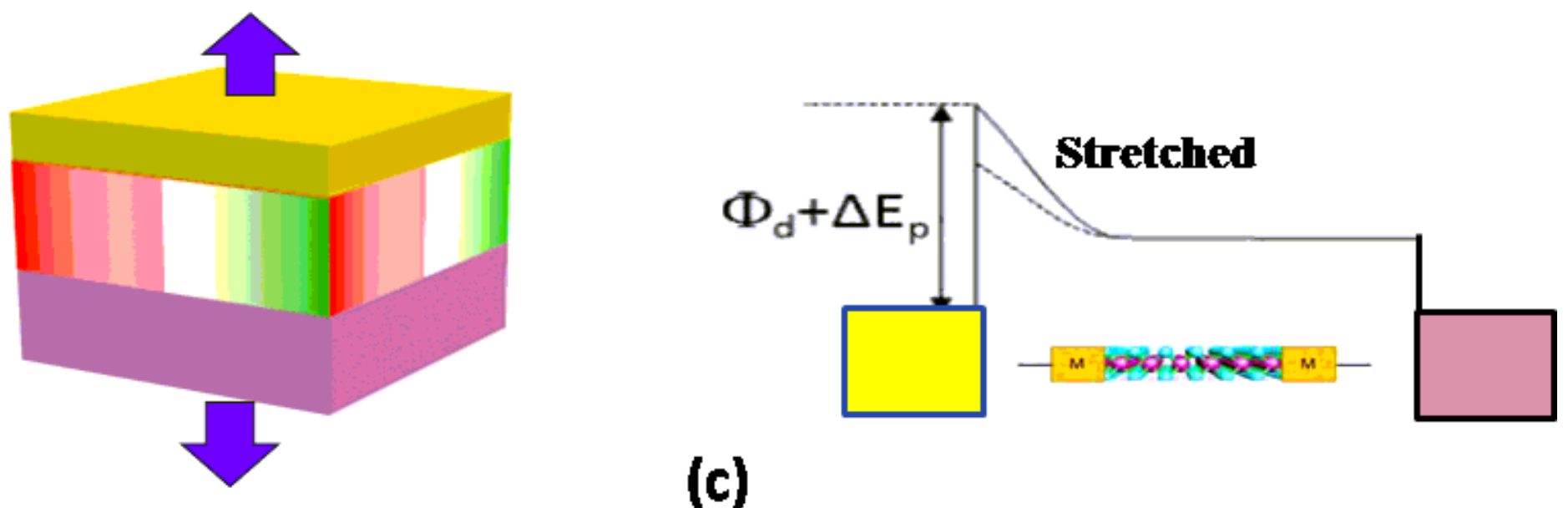

(c)

Figure 1 
(a)

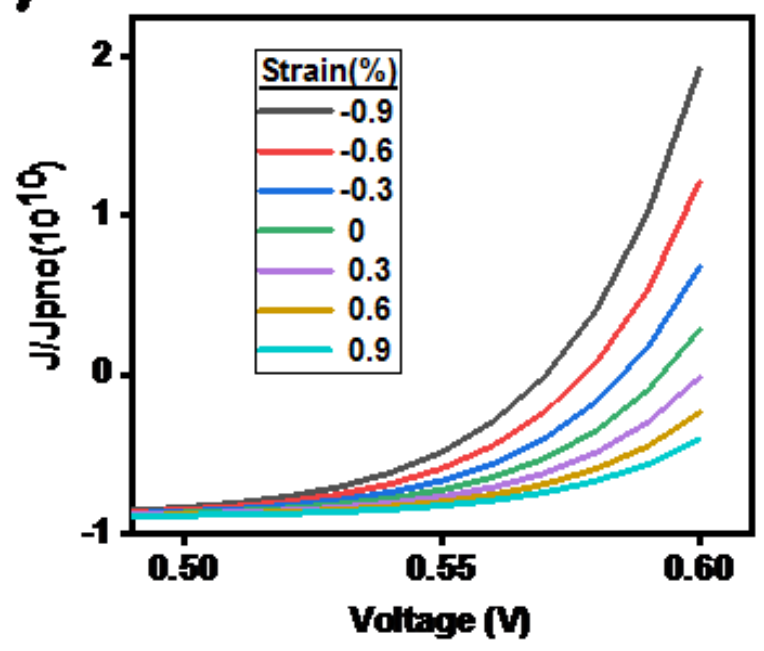

(c)

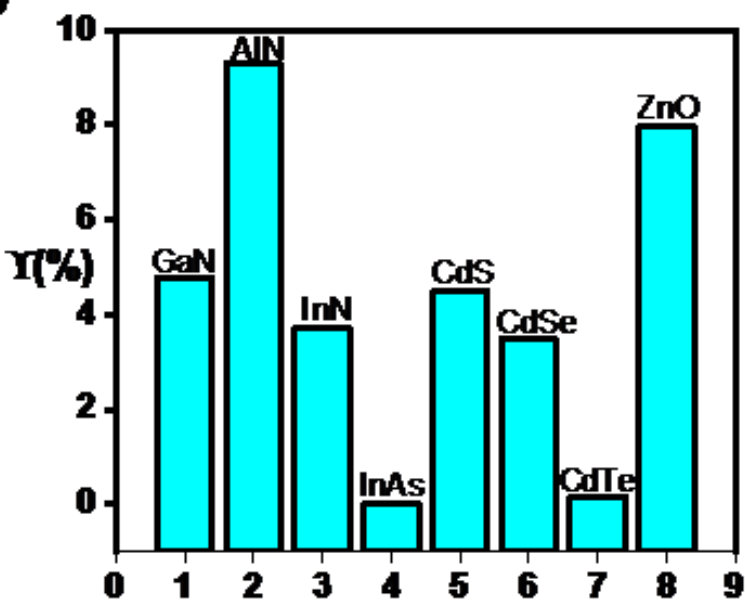

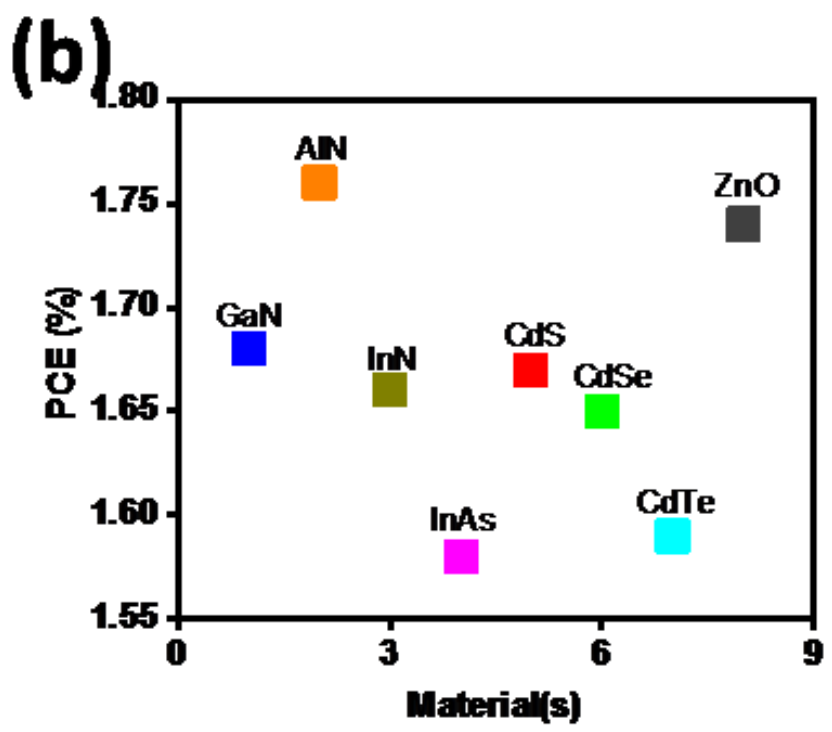

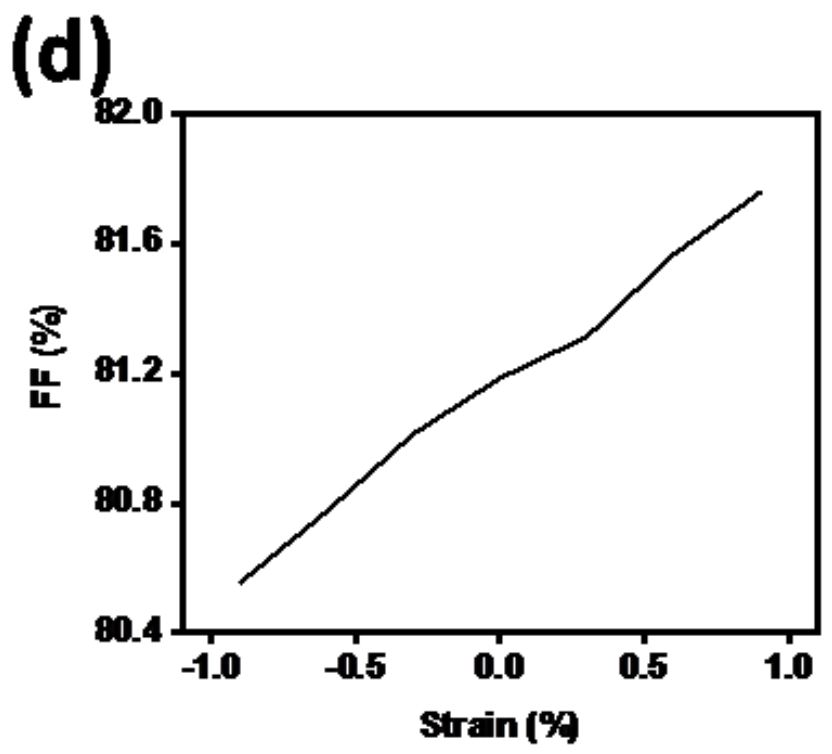

Figure 2 
(a)
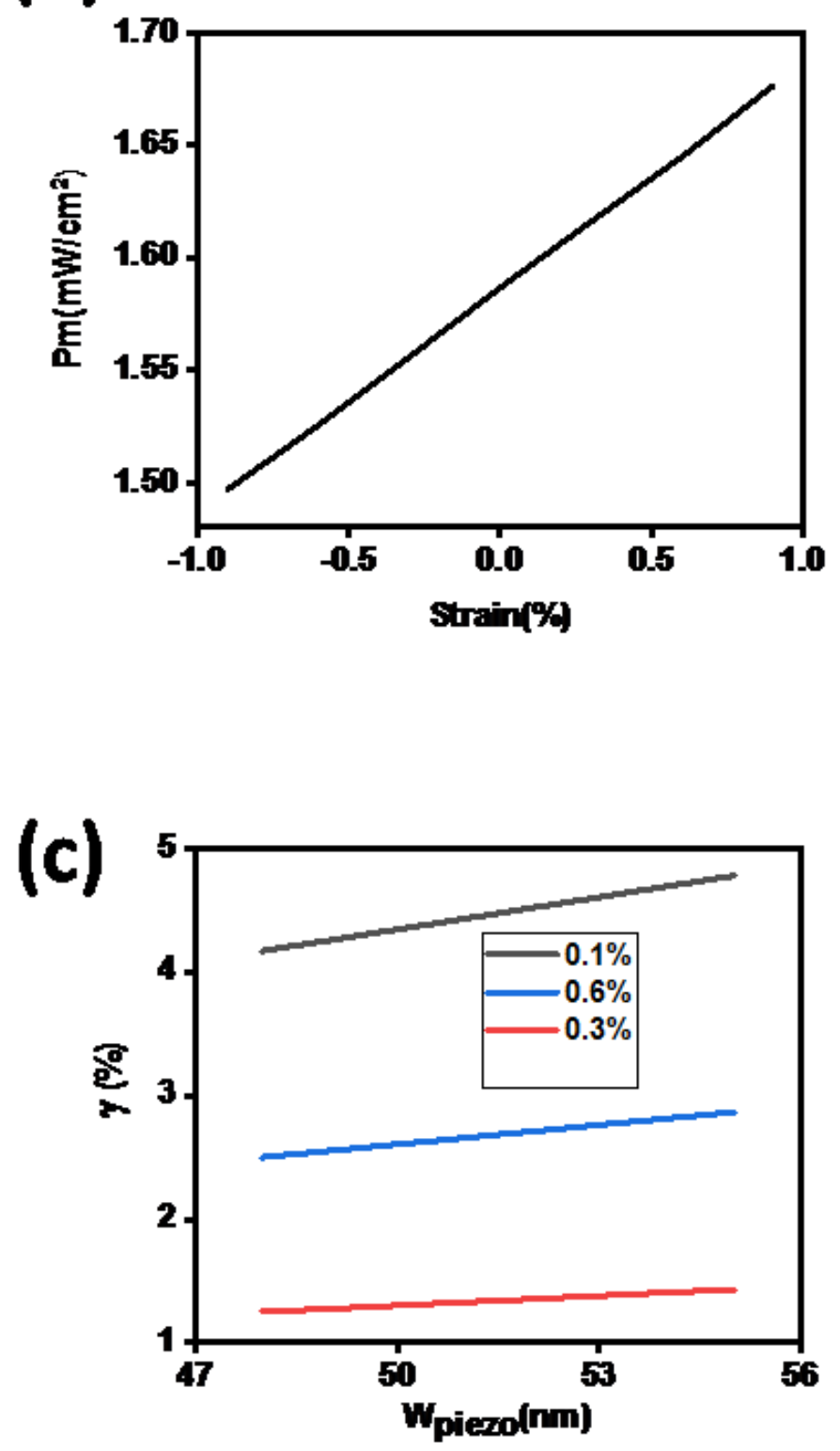

(b)
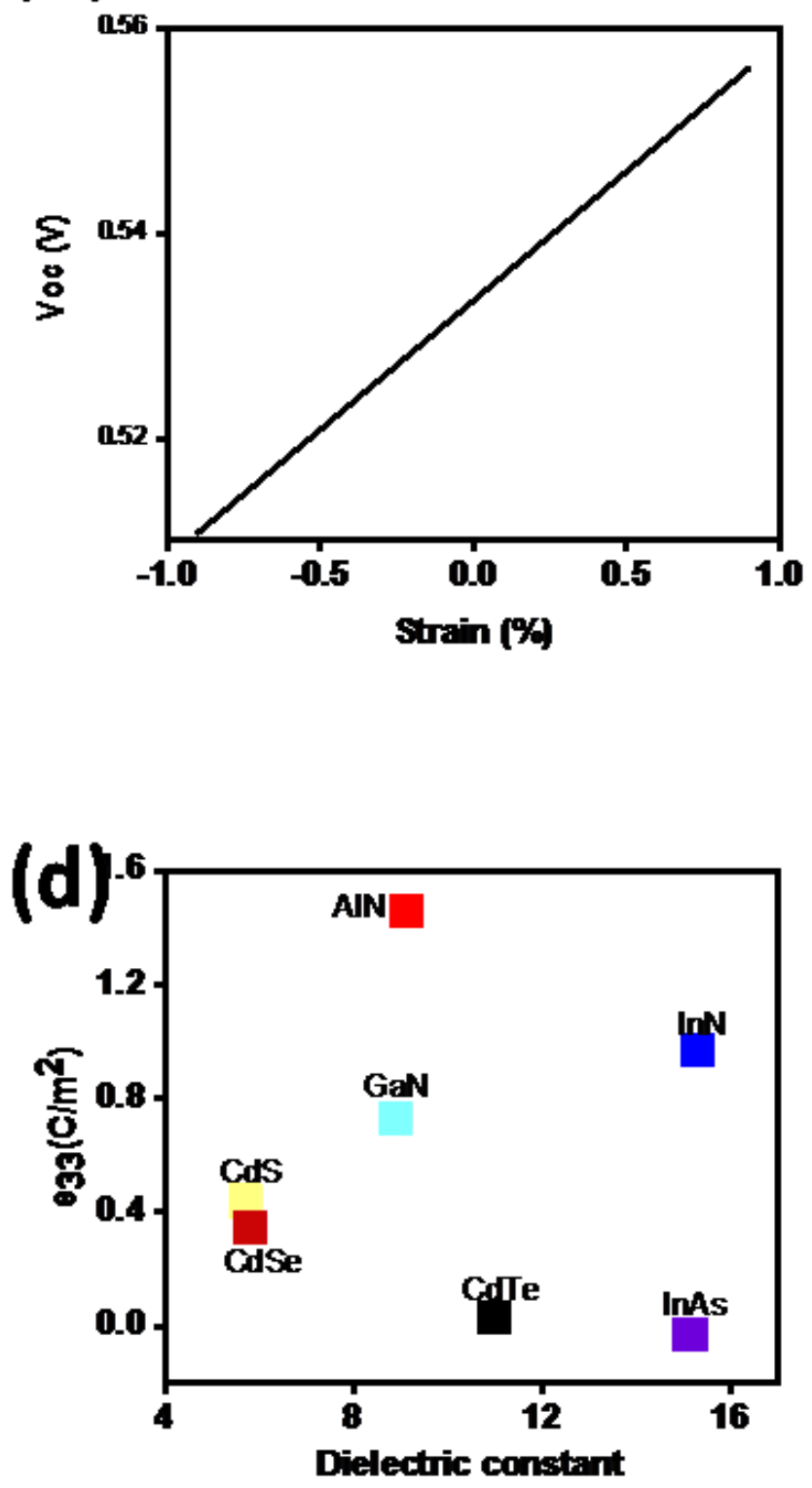

Figure 3

\section{Supplementary Files}

This is a list of supplementary files associated with this preprint. Click to download.

- GraphicalAbstract.tif 